\title{
MeX pipeline for analysis of mobile genetic elements in cancer genome
}

Preeti P. ${ }^{1}$, Robin Sinha ${ }^{1}$, Kamal Rawal ${ }^{1 \#}$

1. Amity Institute of Biotechnology, Amity University Uttar Pradesh, India.

\#Corresponding Author

Email ID: kamal.rawal@gmail.com

Centre for Computational Biology and Bioinformatics, AIB

Amity University, Noida.

\begin{abstract}
Background: Mobile genetic elements (MGEs) comprise a major portion of the human genome and are essential for genetic diversity. These elements are known to have the capability to induce mutations in the human genome. To date, there are several MGE insertions which have been reported to be associated with cancer. We aim to use genome next-generation sequencing data and appropriate bioinformatics tools to accurately identify the insertion sites of MGEs in the human genome.
\end{abstract}

Results: Herein, we introduce the MeX pipeline for the localization and annotation of MGEs in paired-end sequencing data. It requires the reference genome sequence, MGE sequences and paired-end sequencing reads. We evaluated MeX on high depth ( $>75 \times$ ) Illumina HiSeq data produced at the Broad Institute (NA12878) against human genome 38-built (including only chromosome 1, 2 and 3) and Alu elements. We could identify 78 reference and 1 non-reference Alu insertions in the NA12878 sample. Upon annotation, it was found that the non-reference Alu element was in the 3' UTR region of the RNF2 gene. Out of 78 reference insertions, 42 were in the intronic region, 7 in the upstream region, 5 in the downstream region, 1 in the 3 ' UTR region and the rest were not associated with any gene. MeX showed high performance for the identification and annotation of MGEs in genome samples.

Conclusion: This study showed that MeX is a robust and powerful tool for the identification and annotation of MGE insertions. It may also serve as a valuable tool to study the phenotypic changes resulting from transpositional events in cancer genomics.

Keywords: Transposon, paired-end sequencing, cancer, target-site duplication, variant annotation

\section{INTRODUCTION}

MGEs, also known as transposons or jumping genes, are the DNA segments that can change their location within the genome. These MGEs are present in all the organisms, prokaryotes as well as eukaryotes and they make up a large fraction of the human genome that include at least $45 \%$ of the whole genome [Pace et al. 2007]. They are known to have a significant role in genome organization and evolution [Makalowski et al. 2019]. Active transposons are also known to cause mutagenic effects that cause several genetic disorders, like cancer [Jang et al. 2019]. A previous study conducted in our lab has shown that the human genes associated with cancer has significantly higher number of MGEs as compared to the genes that are not 
associated with cancer [Preeti et al. 2021]. We have also presented an efficient computational protocol for MGE insertion site analysis. ELAN, the suite of tools uses standard techniques to identify different MGEs and their distribution on the genome [Rawal et al. 2011]. Previously, in our lab we have also developed efficient platform for vaccine target identification [Rawal et al. 2021] and generated comprehensive maps of molecules that are implicated in human associated diseases [Jagannadham et al. 2016].

Recent advancement in high-throughput sequencing technologies have given opportunities to understand the functionality of transposable elements and their role in cancer. However, due to the repetitive nature of these DNA segments, their identification remains a challenging area of computational biology. Despite of the complexity, there are many tools available for the identification of MGEs, like, RetroSeq [Keane et al. 2013], Tangram [Wu et al. 2014], TraFiC [Tubio et al. 2014], TranspoSeq [Helman et al. 2014], Tea [Lee et al. 2012], TEMP [Zhuang et al. 2014], ngs_te_mapper [Linheiro et al. 2012], PopoolationTE [Kofler et al. 2012], iMGEins [Bae et al 2018], and MGERT [Guliave et al. 2019]. There is no single MGE detection method that can be concluded as best. Nelson et al. 2017 applied a consensus approach, where multiple methods are integrated in a pipeline for the identification.

Being a mutagenic agent, transposons play a role in cancer development. A very important application of such detection methods could be to find the impact of MGE insertion in the human genome and its consequences on cancer development. Here, in this study we generated a framework, named MeX, for MGE insertion identification and their characterization to determine its correlation to cancer.

\section{METHODOLOGY}

\section{Input Requirement}

MeX requires three input files, sequencing reads in fastq format, human reference genome (hg18 or hg19) in fasta format and reference MGEs in fasta format. This pipeline takes paired-end sequencing data as sample input. To evaluate MeX, we used high depth (>75×) Illumina HiSeq data produced at the Broad Institute from the 1000 Genomes Project. Whole genome sequencing of (CEU) Utah residents with ancestry from Northern and Western Europe. This dataset was downloaded from NCBI SRA under accession number SRR622461. The complete human reference genome can be downloaded from UCSC browser for required genome assembly. We extracted the repeat database from Repbase [Bao et al. 2015]. To assess the efficiency of the pipeline a section of the human genome, (including chromosome 1, 2 and 3) and the repeat database (including all the Alu elements) was used.

\section{MeX Implementation}

a. Preprocessing of reads

Fastq file preprocessing is an essential step to obtain clean data for downstream processing. The pipeline utilizes the fastp tool [Chen et al. 2018] for preprocessing. To filter out low quality reads, we performed filtering of reads, pruning of reads, adapter trimming, polyG/polyX tail trimming and UMI preprocessing using a parallel processing approach. It automatically searches for the adapter region in the sequence reads and trim with even only one base in the tail. If fastp identified overlapping regions in paired-end reads, it was automatically corrected, only if one pair had high quality $(\mathrm{Q}>30)$ and another pair had low quality $(\mathrm{Q}<15)$. This step also removed polyG and polyX from read tails as these are common artifacts in Illumina sequencing. While UMI preprocessing, it reduced background noise and improved sensitivity for ultra-low frequency mutation detection. 
b. Quality Control Check

The second step provided a quality control check that was performed with the help of the FastQC tool. It ensured that there were no problems or biases in the preprocessed data. It provided a summary of the total number of sequences processed, lengths of the shortest or longest sequence reads, overall percentage of GC content, sequence quality per base, quality score per sequence, overall $\mathrm{N}$ content, degree of duplication, etc. All these parameters were evaluated and results were represented as pass or fail.

c. MGE Identification

MGE target site identification was performed using ngs_te_mapper2 [Bergman, 2012]. The identification process is detailed as follows:

i. Raw sequence read mapping with MGE database

This step takes two input files, including sequencing reads (in fastq) and MGE database (in fasta). First, all the reads were aligned to the MGE database. After alignment, those reads were extracted whose match includes the start or end of the query MGE. If a read had two or more matches to different MGEs then the best match was selected. The best match was categorized based on the length of the matching sequence and the quality of the matched sequence. The quality was decided based on the number of gaps and mismatches along with the lower number of blocks. Those read matches with more than 1 block, mismatch, or gap for every 20 bp of target and query sequences were rejected.

ii. Selected reads mapping with reference genome

Those reads that had aligned with either at the start or end of MGEs, were mapped to reference the human genome. We then selected for mapped reads with one or fewer mismatches in 20 for both the read and reference genome sequences. Reads were retained if a match to the reference genome or TE was included at the beginning or end of the read. These sequences also had to match the reference TE start/end exactly where the genomic region begins or vice versa.

iii. Identification of target site duplication (TSD)

To find TSDs, mapped reads that had distances between the start coordinate of one read and the end coordinate of the next read were found sequentially in the genome that overlapped by less than or equal to $20 \mathrm{bp}$. This overlap distance is defined as TSD. The identified MGEs are then reported in bed format with information regarding chromosome number, start and end position, identified transposon family, and sense of the identified strand.

\section{Variant Calling}

The non-reference MGE insertions were then called as variants in Variant Call File (VCF) format. This file comprises information of chromosomal number, genomic location, transposon family, and supporting reads. All this information is then used for annotation of the identified MGE insertion.

\section{Annotation}


The VCF annotation was performed using the Ensembl Variant Effect Predictor (VEP) [McLaren et al. 2016]. This annotation step will predict multiple features of the identified non-reference MGE. MeX provides user-defined options for annotation with respect to GRCh37 and GRCh38 assembly. It provides all the information regarding, gene, transcript ID, predicted consequence, nucleotide change at coding region, amino acid change at protein level, exon/intron number, and prediction of impact on protein function using in silico tools like SIFT [Ng et al. 2003] and Polyphen [Adzhubei et al. 2010]. MGE at a single position were initially annotated with respect to all different transcripts for the given gene. However, it was further filtered to only canonical transcript annotation.

\section{RESULTS}

\section{MeX implementation}

a. Preprocessing

Figure 2 represents the general summary of this step. $31.30 \mathrm{M}$ reads were removed to enhance the overall quality of the sample. The base calling accuracy, measured by Phred quality score (represented as Q score), was increased from $84.45 \%$ to $96.41 \%$ at Q score 20 and from $80.60 \%$ to $93.16 \%$ at Q score 30 . There was no significant difference in GC content. The overall rate of duplication is $5.34 \%$ and $98.59 \%$ of reads are with unknown length of insert size (see Figure 3 and 4). Figure 5 and 6 shows the difference in the comparison of read quality and base content before and after preprocessing.

b. Quality check

All the quality parameters of read 1 and read 2 after the preprocessing are represented in Figure 7 and 8. All the parameters passed the qualification criteria, except for per sequence GC content and sequence length distribution, which showed medium qualification. Overall the sample was qualified for further evaluation.

c. MGE Identification

Using the MeX pipeline we could identify 78 reference and 1 non-reference Alu elements in the NA12878 sample in the first three chromosomes (see Table S1 and Table S2).

\section{Variant Calling and Annotation}

All the identified MGEs were called in BED file format and these transposons were converted to VCF files/formats. These transposons were then annotated. The reference elements were identified in 55 different genes (see Table S3). Out of these 55 reference elements, 42 are in the intronic region, 7 in the upstream region, 5 in the downstream region, and 1 in the 3' UTR region. No reference transposon was identified in the exonic region. The non-reference element was identified in the RNF2 gene (see Table 3). The RNF2 gene element was annotated to be in the 3' UTR region.

\section{CONCLUSION}

MGEs play an important role in genomic instability. Recent studies have shown that MGE insertion can cause mutagenic changes to the human genome that leads to phenotypic changes like cancer. This suggests the importance of the dynamics of MGEs in the human genome. MeX was developed to accurately identify the breakpoints of inserted MGEs and predict the possible changes that will be caused by the inserted MGE 
at protein level. MeX has a valuable feature of annotating the identified MGEs that makes it better than the other available MGE identification tools.

\section{Contribution of Authors}

This study was conducted under the overall guidance of KR, who contributed in protocol, critical evaluation of data and manuscript. The pipeline was designed, constructed and validated by PP and RS. Manuscript writing was done by PP and KR. All the authors are responsible for the content of the manuscript.

\section{Acknowledgement}

We extend our sincere gratitude to Amity University for providing administrative and technical support required in the conduct of this study.

\section{Financial Support and Sponsorship}

Dr. Kamal Rawal acknowledges the support provided by DBT, Government of India for the grant of the Department of Biotechnology, Ministry of Science and Technology, Government of India (Grant Id: BT/PRI7252/BID/7/708/2016) and SERB, Department of Science Technology (File Number: CVD/2020/000842). Robin Sinha was supported by a Grant from the Robert J. Kleberg, Jr. and Helen C. Kleberg Foundation, USA, Texas Children's Hospital and Baylor College of Medicine, Houston USA.

\section{REFERENCES}

1. Pace JK 2nd, Feschotte C. The evolutionary history of human DNA transposons: evidence for intense activity in the primate lineage. Genome Res. 2007 Apr;17(4):422-32. doi: 10.1101/gr.5826307. Epub 2007 Mar 5. PMID: 17339369; PMCID: PMC1832089.

2. Makałowski W, Gotea V, Pande A, Makałowska I. Transposable Elements: Classification, Identification, and Their Use As a Tool For Comparative Genomics. Methods Mol Biol. 2019;1910:177-207. doi: 10.1007/978-1-4939-9074-0_6. PMID: 31278665.

3. Jang HS, Shah NM, Du AY, Dailey ZZ, Pehrsson EC, Godoy PM, Zhang D, Li D, Xing X, Kim S, O'Donnell D, Gordon JI, Wang T. Transposable elements drive widespread expression of oncogenes in human cancers. Nat Genet. 2019 Apr;51(4):611-617. doi: 10.1038/s41588-019-0373-3. Epub 2019 Mar 29. Erratum in: Nat Genet. 2019 May;51(5):920. PMID: 30926969; PMCID: PMC6443099.

4. P. Preeti, Tapan K. Baral, and Kamal Rawal. 2021. "DISTRIBUTION OF MOBILE GENETIC ELEMENTS IN CANCER GENES.” OSF Preprints. January 14. doi:10.31219/osf.io/8b4j3

5. Rawal K, Ramaswamy R. Genome-wide analysis of mobile genetic element insertion sites. Nucleic Acids Res. 2011 Sep 1;39(16):6864-78. doi: 10.1093/nar/gkr337. Epub 2011 May 23. PMID: 21609951; PMCID: PMC3167599.

6. Jagannadham J, Jaiswal HK, Agrawal S, Rawal K. Comprehensive Map of Molecules Implicated in Obesity. PLoS One. 2016 Feb 17;11(2):e0146759. doi: 10.1371/journal.pone.0146759. PMID: 26886906; PMCID: PMC4757102.

7. Keane TM, Wong K, Adams DJ. RetroSeq: transposable element discovery from next-generation sequencing data. Bioinformatics. 2013 Feb 1;29(3):389-90. doi: 10.1093/bioinformatics/bts697. Epub 2012 Dec 10. PMID: 23233656; PMCID: PMC3562067. 
8. Wu J, Lee WP, Ward A, Walker JA, Konkel MK, Batzer MA, Marth GT. Tangram: a comprehensive toolbox for mobile element insertion detection. BMC Genomics. 2014 Sep 16;15(1):795. doi: 10.1186/1471-2164-15-795. PMID: 25228379; PMCID: PMC4180832.

9. Tubio JMC, Li Y, Ju YS, Martincorena I, Cooke SL, Tojo M, Gundem G, Pipinikas CP, Zamora J, Raine K, Menzies A, Roman-Garcia P, Fullam A, Gerstung M, Shlien A, Tarpey PS, Papaemmanuil E, Knappskog S, Van Loo P, Ramakrishna M, Davies HR, Marshall J, Wedge DC, Teague JW, Butler AP, Nik-Zainal S, Alexandrov L, Behjati S, Yates LR, Bolli N, Mudie L, Hardy C, Martin S, McLaren S, O'Meara S, Anderson E, Maddison M, Gamble S, Foster C, Warren AY, Whitaker H, Brewer D, Eeles R, Cooper C, Neal D, Lynch AG, Visakorpi T, Isaacs WB, Veer LV, Caldas C, Desmedt C, Sotiriou C, Aparicio S, Foekens JA, Eyfjörd JE, Lakhani SR, Thomas G, Myklebost O, Span PN, Børresen-Dale AL, Richardson AL, Van de Vijver M, Vincent-Salomon A, Van den Eynden GG, Flanagan AM, Futreal PA, Janes SM, Bova GS, Stratton MR, McDermott U, Campbell PJ; ICGC Breast Cancer Group; ICGC Bone Cancer Group; ICGC Prostate Cancer Group. Mobile DNA in cancer. Extensive transduction of nonrepetitive DNA mediated by L1 retrotransposition in cancer genomes. Science. 2014 Aug 1;345(6196):1251343. doi: 10.1126/science.1251343. PMID: 25082706; PMCID: PMC4380235.

10. Helman E, Lawrence MS, Stewart C, Sougnez C, Getz G, Meyerson M. Somatic retrotransposition in human cancer revealed by whole-genome and exome sequencing. Genome Res. 2014 Jul;24(7):105363. doi: 10.1101/gr.163659.113. Epub 2014 May 13. PMID: 24823667; PMCID: PMC4079962.

11. Lee E, Iskow R, Yang L, Gokcumen O, Haseley P, Luquette LJ 3rd, Lohr JG, Harris CC, Ding L, Wilson RK, Wheeler DA, Gibbs RA, Kucherlapati R, Lee C, Kharchenko PV, Park PJ; Cancer Genome Atlas Research Network. Landscape of somatic retrotransposition in human cancers. Science. 2012 Aug 24;337(6097):967-71. doi: 10.1126/science.1222077. Epub 2012 Jun 28. PMID: 22745252; PMCID: PMC3656569.

12. Zhuang J, Wang J, Theurkauf W, Weng Z. TEMP: a computational method for analyzing transposable element polymorphism in populations. Nucleic Acids Res. 2014 Jun;42(11):6826-38. doi: 10.1093/nar/gku323. Epub 2014 Apr 21. PMID: 24753423; PMCID: PMC4066757.

13. Linheiro RS, Bergman CM. Whole genome resequencing reveals natural target site preferences of transposable elements in Drosophila melanogaster. PLoS One. 2012;7(2):e30008. doi: 10.1371/journal.pone.0030008. Epub 2012 Feb 9. PMID: 22347367; PMCID: PMC3276498.

14. Kofler R, Betancourt AJ, Schlötterer C. Sequencing of pooled DNA samples (Pool-Seq) uncovers complex dynamics of transposable element insertions in Drosophila melanogaster. PLoS Genet. 2012 Jan;8(1):e1002487. doi: 10.1371/journal.pgen.1002487. Epub 2012 Jan 26. PMID: 22291611; PMCID: PMC3266889.

15. Bae J, Lee KW, Islam MN, Yim HS, Park H, Rho M. iMGEins: detecting novel mobile genetic elements inserted in individual genomes. BMC Genomics. 2018 Dec 18;19(1):944. doi: 10.1186/s12864-018-5290-9. PMID: 30563451; PMCID: PMC6299635.

16. Guliaev AS, Semyenova SK. MGERT: a pipeline to retrieve coding sequences of mobile genetic elements from genome assemblies. Mob DNA. 2019 May 14;10:21. doi: 10.1186/s13100-019-0163-6. PMID: 31114637; PMCID: PMC6515669.

17. Nelson MG, Linheiro RS, Bergman CM. McClintock: An Integrated Pipeline for Detecting Transposable Element Insertions in Whole-Genome Shotgun Sequencing Data. G3 (Bethesda). 2017 Aug 7;7(8):2763-2778. doi: 10.1534/g3.117.043893. PMID: 28637810; PMCID: PMC5555480. 
18. Chen S, Zhou Y, Chen Y, Gu J. fastp: an ultra-fast all-in-one FASTQ preprocessor. Bioinformatics. 2018 Sep 1;34(17):i884-i890. doi: 10.1093/bioinformatics/bty560. PMID: 30423086; PMCID: PMC6129281.

19. Andrews, S. FastQC: a quality control tool for high throughput sequence data. 2010.

20. Bergman CM. A proposal for the reference-based annotation of de novo transposable element insertions. Mob Genet Elements. 2012 Jan 1;2(1):51-54. doi: 10.4161/mge.19479. PMID: 22754753; PMCID: PMC3383450.

21. Bao W, Kojima KK, Kohany O. Repbase Update, a database of repetitive elements in eukaryotic genomes. Mob DNA. 2015 Jun 2;6:11. doi: 10.1186/s13100-015-0041-9. PMID: 26045719; PMCID: PMC4455052.

22. McLaren W, Gil L, Hunt SE, Riat HS, Ritchie GR, Thormann A, Flicek P, Cunningham F. The Ensembl Variant Effect Predictor. Genome Biol. 2016 Jun 6;17(1):122. doi: 10.1186/s13059-0160974-4. PMID: 27268795; PMCID: PMC4893825.

23. Ng PC, Henikoff S. SIFT: Predicting amino acid changes that affect protein function. Nucleic Acids Res. 2003 Jul 1;31(13):3812-4. doi: 10.1093/nar/gkg509. PMID: 12824425; PMCID: PMC168916.

24. Adzhubei IA, Schmidt S, Peshkin L, Ramensky VE, Gerasimova A, Bork P, Kondrashov AS, Sunyaev SR. A method and server for predicting damaging missense mutations. Nat Methods. 2010 Apr;7(4):248-9. doi: 10.1038/nmeth0410-248. PMID: 20354512; PMCID: PMC2855889. 
Figure 1. Overview of MeX pipeline

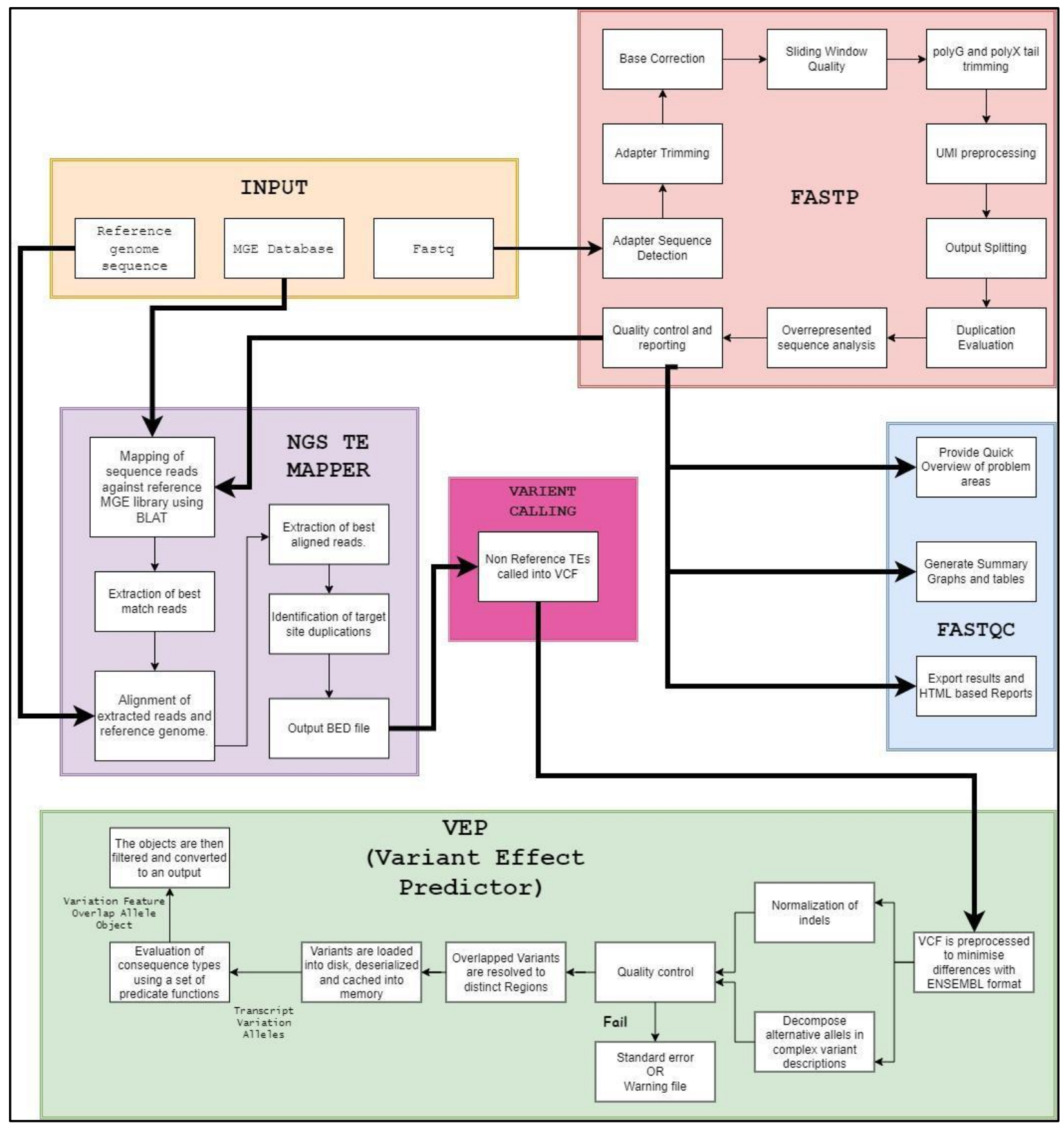


Figure 2. Summary of Preprocessing Step

\begin{tabular}{|c|c|c|}
\hline \multicolumn{3}{|l|}{ General } \\
\hline fastp version: & \multicolumn{2}{|c|}{0.20 .1 (https://github. com/OpenGene/fastp) } \\
\hline sequencing: & \multicolumn{2}{|c|}{ paired end (101 cycles + 101 cycles) } \\
\hline mean length before filtering: & \multicolumn{2}{|c|}{$101 \mathrm{bp}, 101 \mathrm{bp}$} \\
\hline mean length after filtering: & \multicolumn{2}{|l|}{$100 \mathrm{bp}, 100 \mathrm{bp}$} \\
\hline duplication rate: & \multicolumn{2}{|l|}{5.3441088} \\
\hline Insert size peak: & \multicolumn{2}{|l|}{31} \\
\hline \multicolumn{3}{|l|}{ Before filtering } \\
\hline total reads: & \multicolumn{2}{|l|}{$179.965424 \mathrm{M}$} \\
\hline total bases: & \multicolumn{2}{|l|}{$18.176508 \mathrm{G}$} \\
\hline Q20 bases: & \multicolumn{2}{|l|}{15.349548 G $(84.4471818)$} \\
\hline Q30 bases: & \multicolumn{2}{|l|}{$14.650156 G(80.5994008)$} \\
\hline GC content: & \multicolumn{2}{|l|}{39.8948258} \\
\hline \multicolumn{3}{|l|}{ After filtering } \\
\hline total reads: & \multicolumn{2}{|l|}{$148.665340 \mathrm{M}$} \\
\hline total bases: & \multicolumn{2}{|l|}{$14.949997 \mathrm{G}$} \\
\hline Q20 bases: & \multicolumn{2}{|l|}{14.412864 G $(96.4071354)$} \\
\hline Q30 bases: & \multicolumn{2}{|l|}{13.927247 G $(93.1588654)$} \\
\hline GC content: & \multicolumn{2}{|l|}{39.3491108} \\
\hline \multicolumn{3}{|l|}{ Filtering result } \\
\hline reads passed filters: & \multicolumn{2}{|l|}{$148.665340 \mathrm{M}(82.6077248)$} \\
\hline reads with low quality: & \multicolumn{2}{|l|}{$30.797632 \mathrm{M}(17.1130838)$} \\
\hline reads with too many $\mathrm{N}$ : & \multicolumn{2}{|l|}{$502.452000 \mathrm{~K}(0.2791948)$} \\
\hline reads too short: & \multicolumn{2}{|l|}{$0(0.0000008)$} \\
\hline \multicolumn{3}{|l|}{ Adapters } \\
\hline \multicolumn{3}{|c|}{$\begin{array}{l}\text { Adapter or bad ligation of read1 } \\
\text { The input has little adopter percentage }(\sim 0.376446\}) \text {, probably it's trimmed before. }\end{array}$} \\
\hline \multicolumn{2}{|l|}{ Sequence } & Occurrences \\
\hline \multicolumn{2}{|l|}{ al1 adapter sequences } & 770722 \\
\hline \multicolumn{3}{|c|}{$\begin{array}{l}\text { Adapter or bad ligation of read2 } \\
\text { The input hos little adapter percentage }(\sim 0.3764464) \text {, probably it's trimmed before. }\end{array}$} \\
\hline \multicolumn{2}{|l|}{ Sequence } & Occurrences \\
\hline \multicolumn{2}{|l|}{ al1 adapter sequences } & 770722 \\
\hline
\end{tabular}


Figure 3. Rate of duplication estimated by Fastp in raw reads. The duplication rate is the fraction of mapped reads where any 2 reads share the same $5^{\prime}$ and $3^{\prime}$ coordinates. $x$-axis represents the percentage of reads and the GC ratio and $y$-axis represents level of duplication (unit unknown)

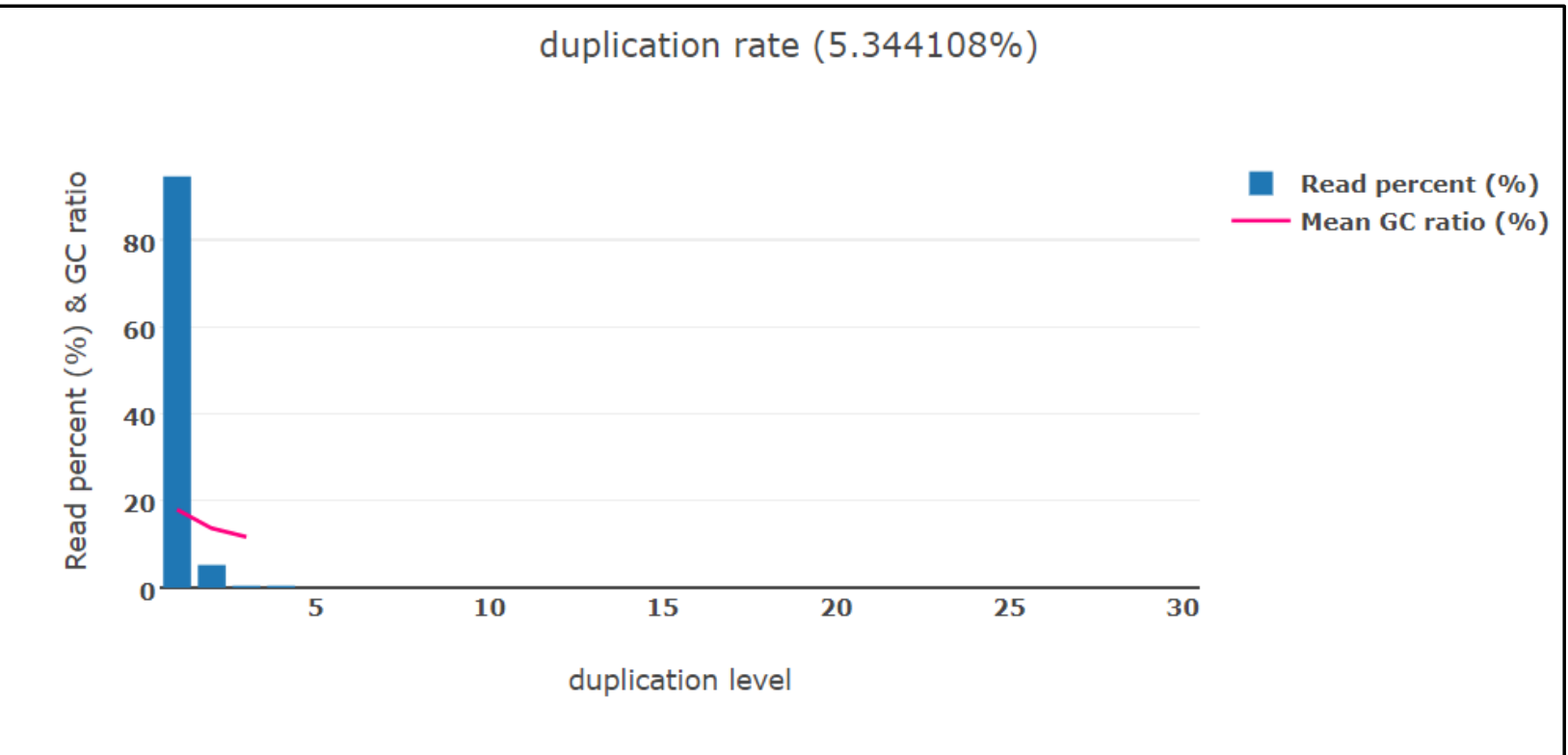

Figure 4. Estimation of insert size by Fastp in raw reads. The insert size is the number of base pairs sequenced from a DNA fragment. $x$-axis represents the percentage of reads and $y$-axis represents insert size (in bp).

Insert size distribution ( $98.589446 \%$ reads are with unknown length)

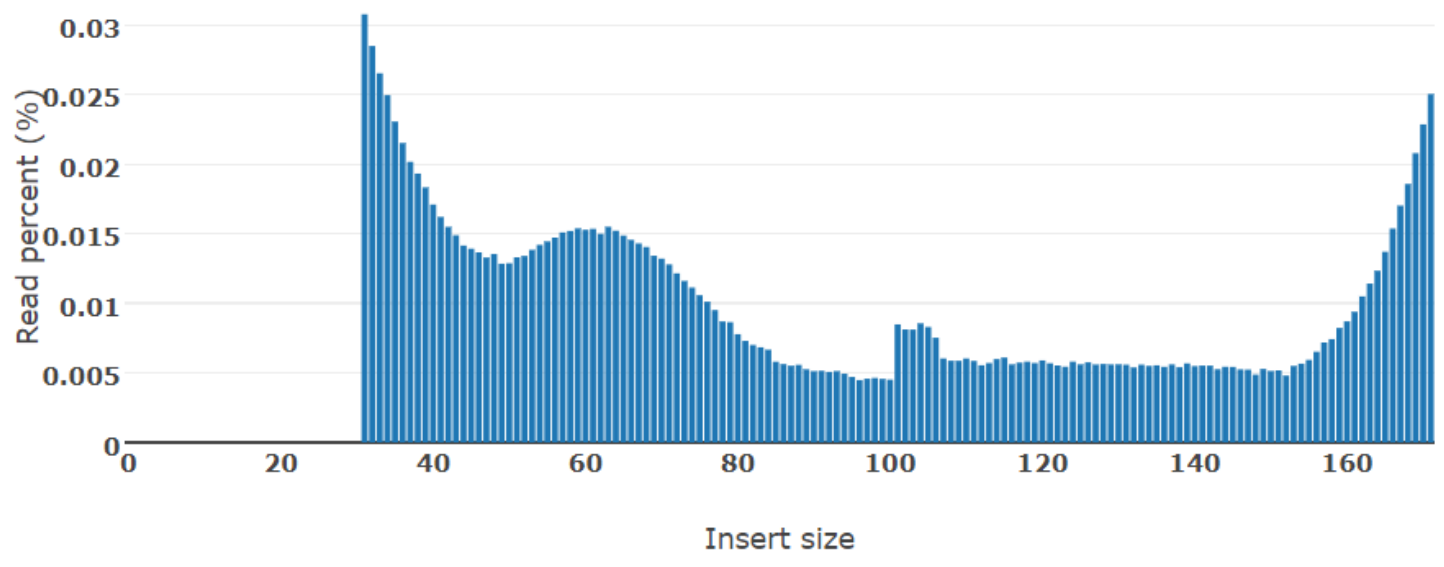

This estimation is based on paired-end overlap analysis, and there are $98.589446 \%$ reads found not overlapped.

The nonoverlapped read pairs may have insert size $<30$ or $>172$, or contain too much sequencing errors to be detected as overlapped. 
Figure 5. Comparison of read quality before and after preprocessing

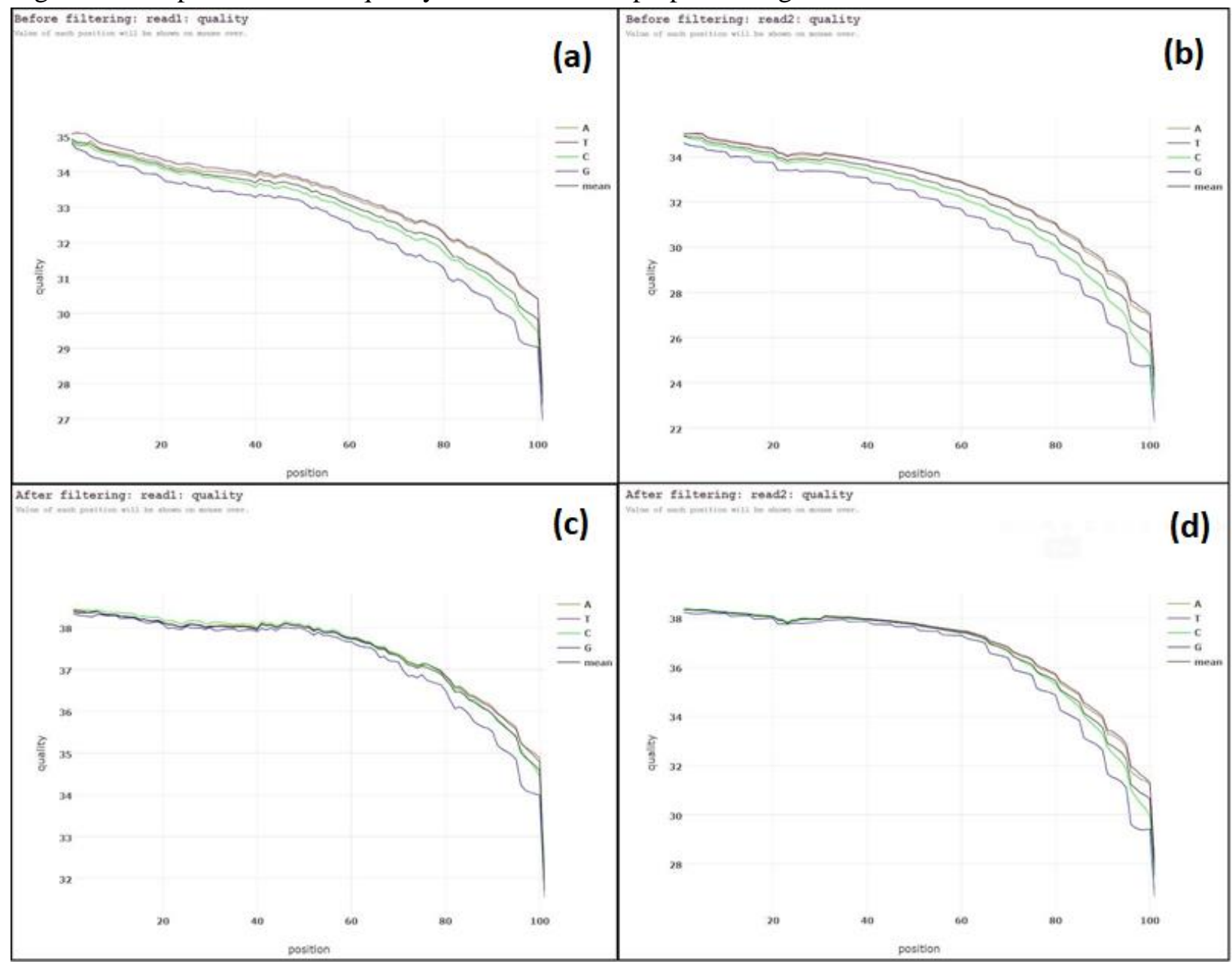


Figure 6: Comparison of base content before and after filtering

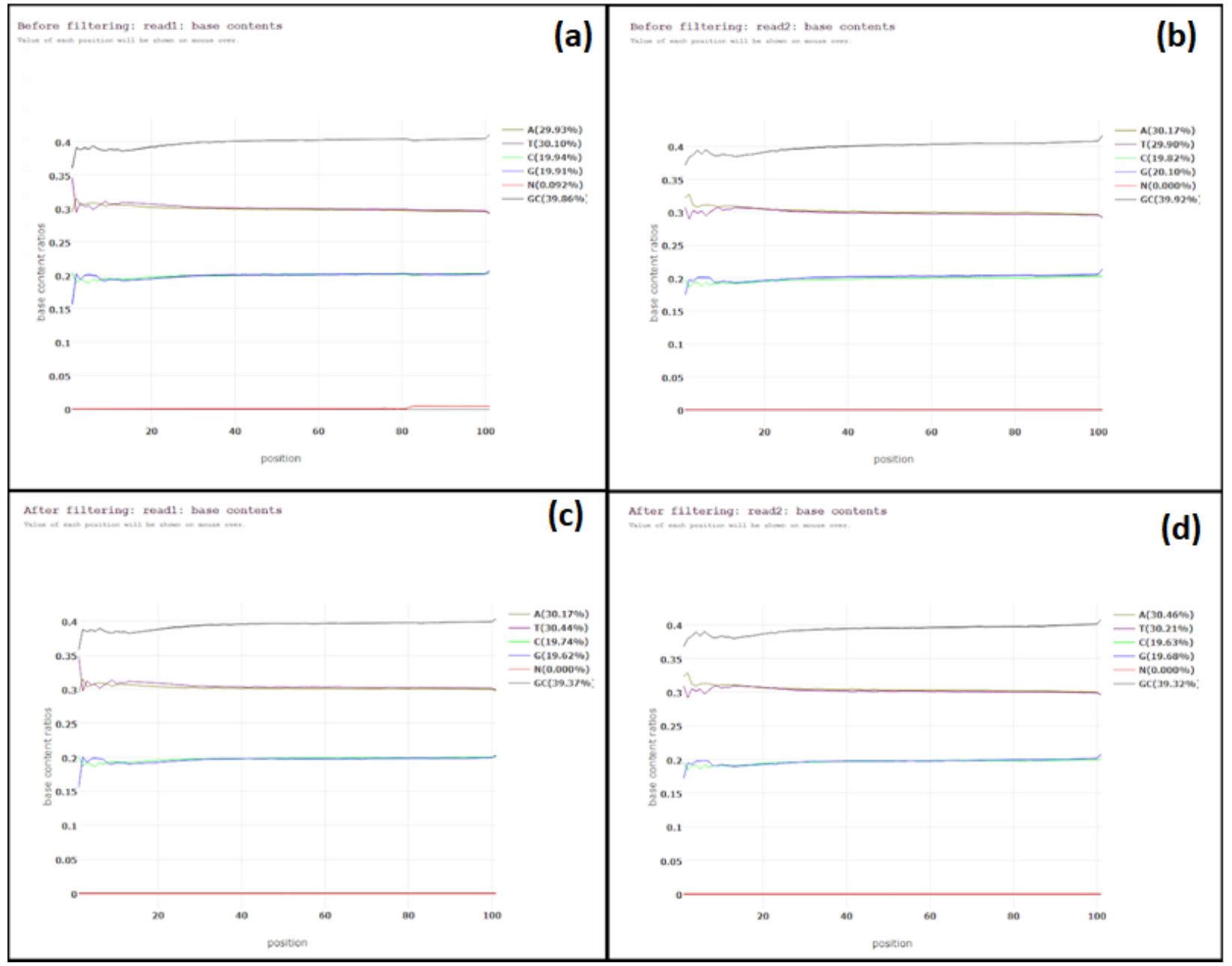


Figure 7. Quality parameters of SRR622461_1

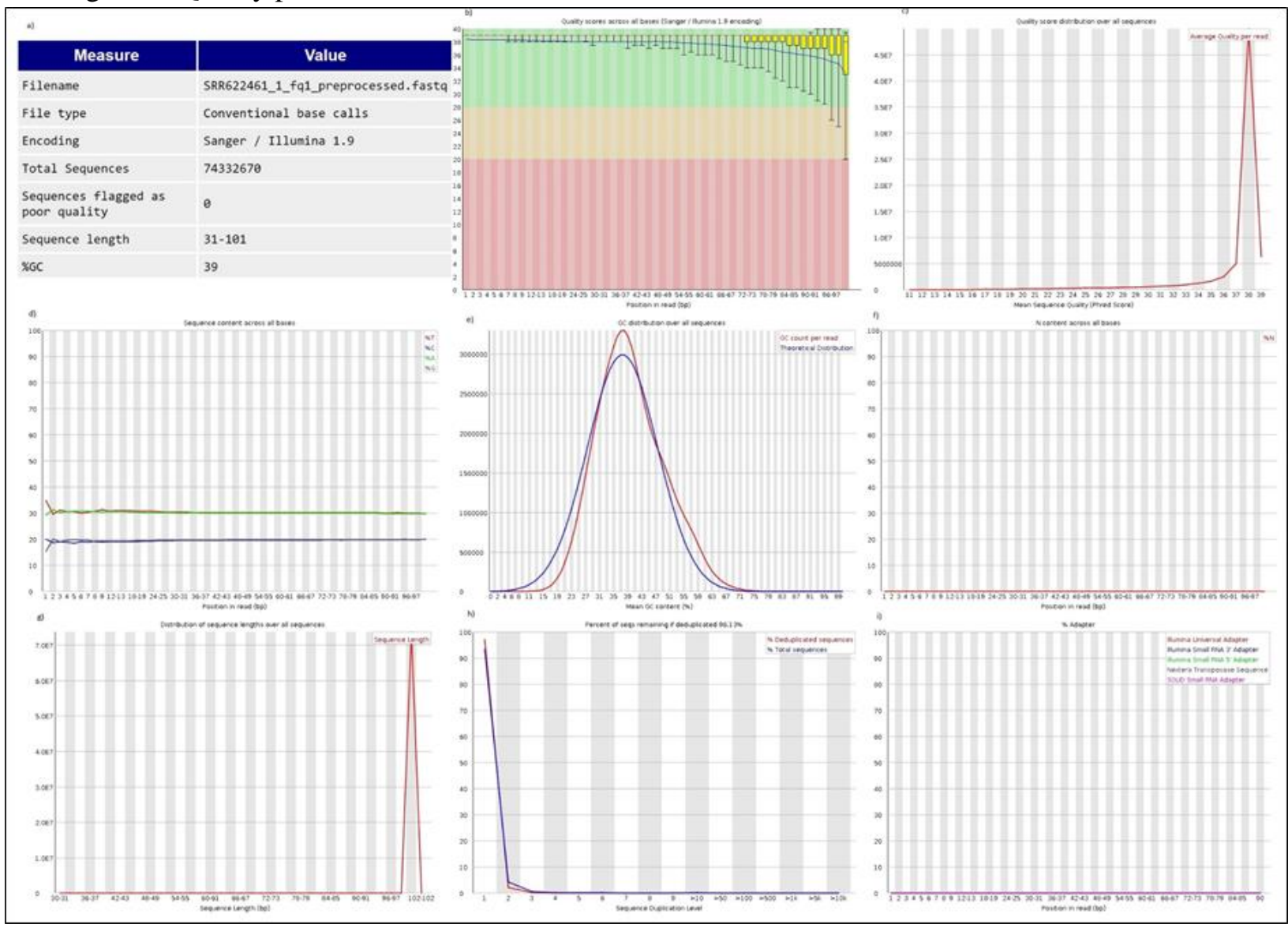


Figure 8. Quality parameters of SRR622461_2

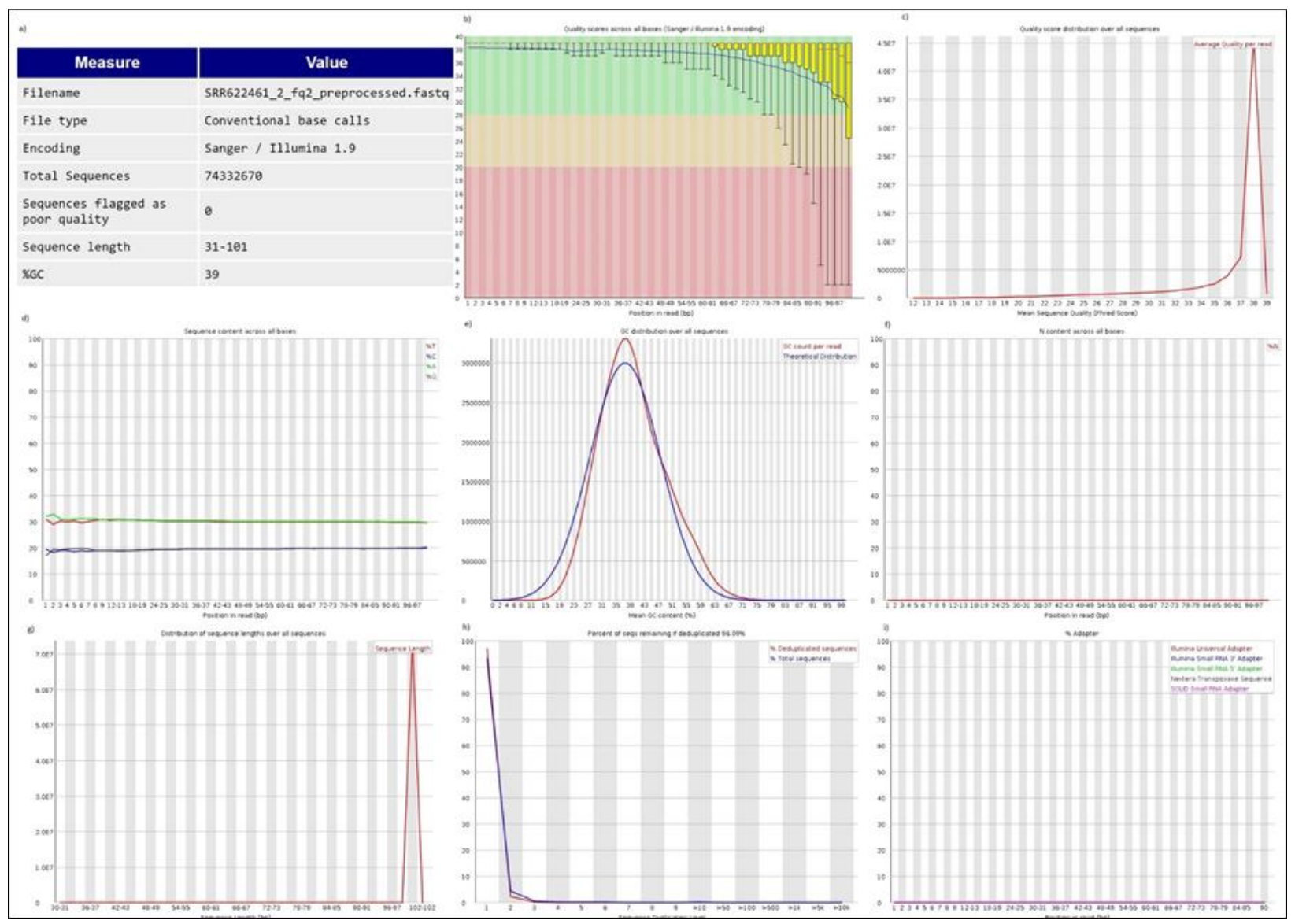


Figure 9:

a) Read Mapping with MGE library

Transposable Element

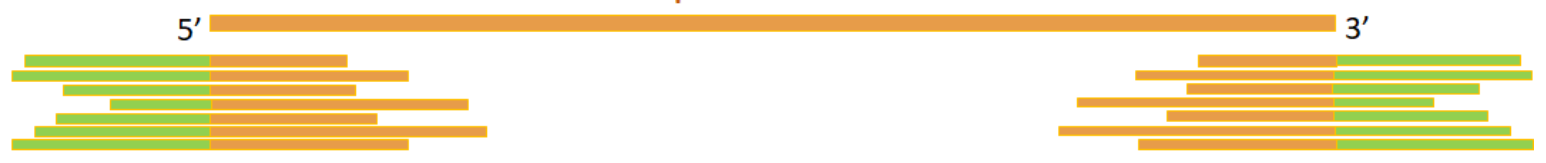

b) Shortlisted read mapping with reference genome

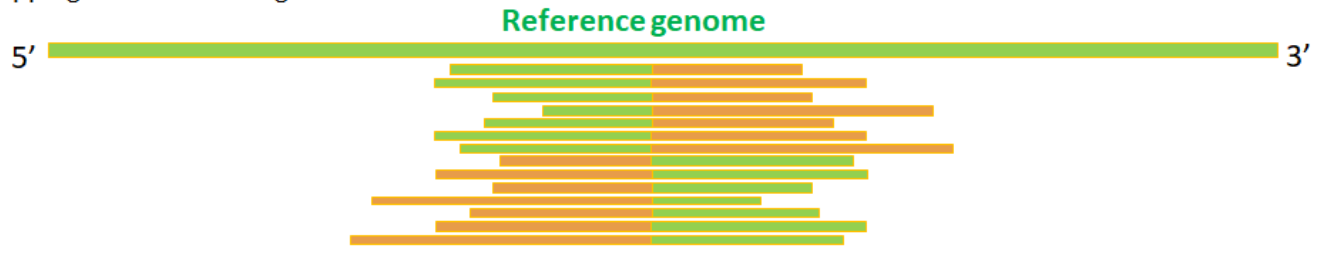

c) Identification of Target-Site Duplication (TSD)

Reference genome

$5^{\prime}$

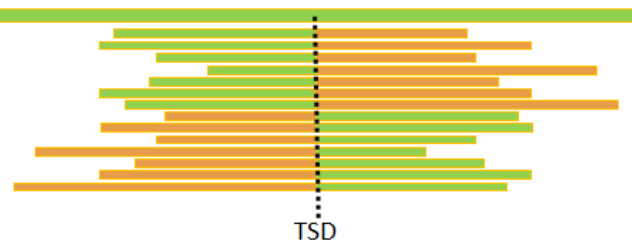

Figure 10:

\section{Statistics}

If the workflow has been executed in cluster/cloud, runtimes include the waiting time in the queue.

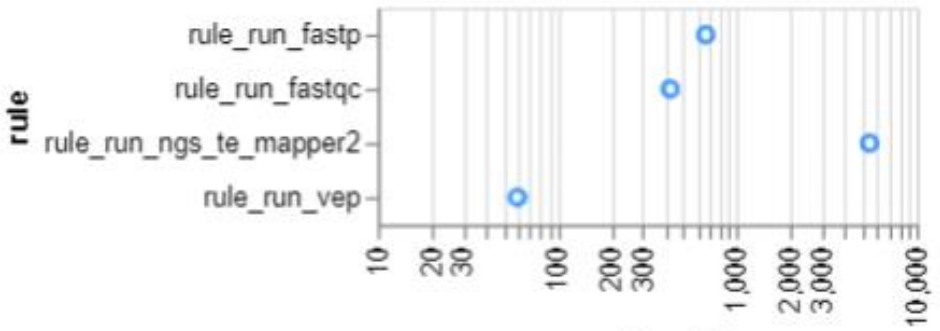

runtime [s]

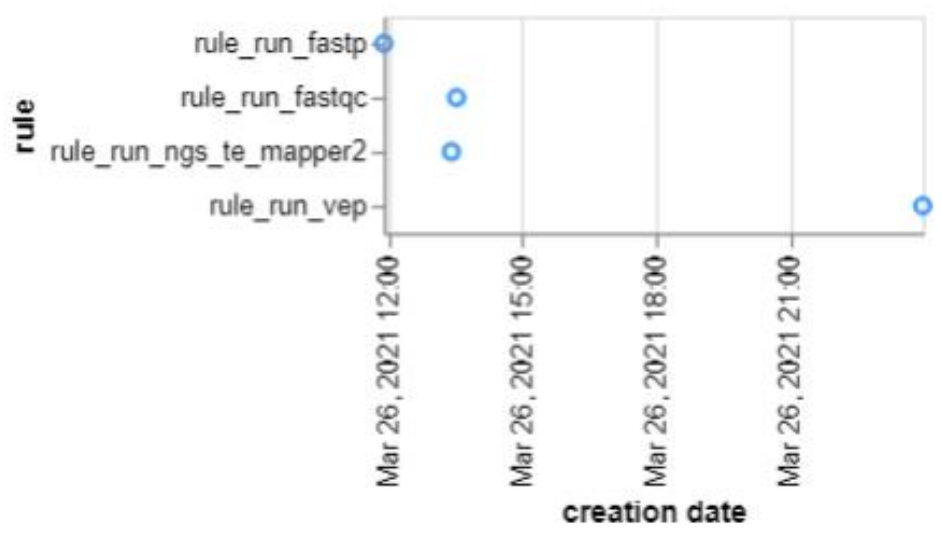


Figure 11:

a)

\section{General statistics}

\begin{tabular}{l|l} 
Lines of input read & 117
\end{tabular}

Variants processed

117

Variants filtered out

Novel / existing variants

Overlapped genes

Overlapped transcripts

Overlapped regulatory features
0

98

904

16 b)

\section{General statistics}

Lines of input read

Variants processed 1

Variants filtered out 0

Novel / existing variants

Overlapped genes 1

Overlapped transcripts 4

Overlapped regulatory features

Figure 12:

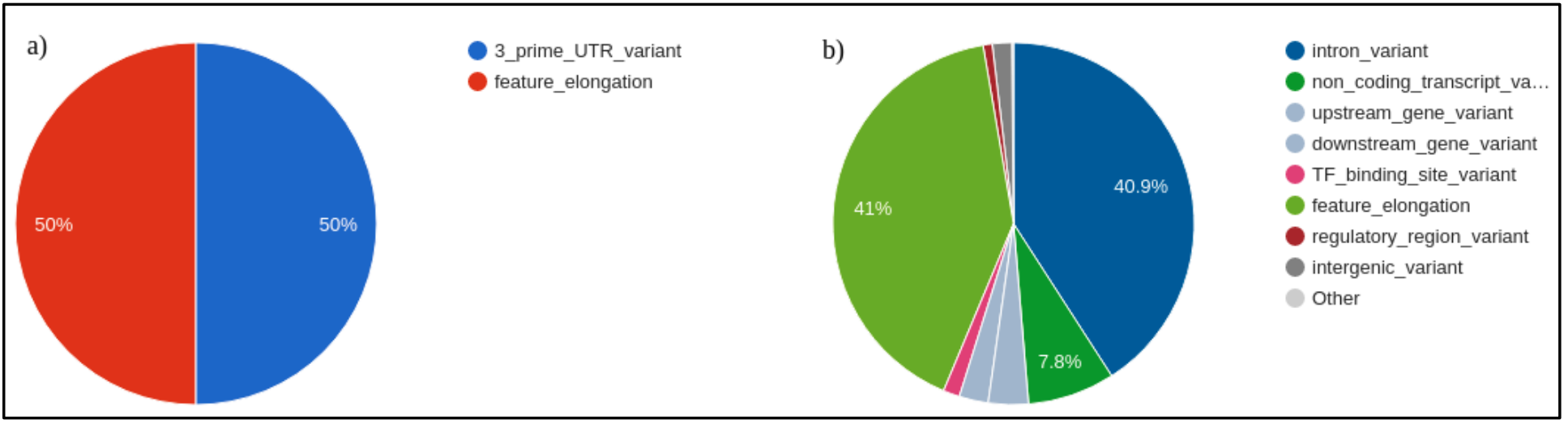


Figure 13:

Variants by chromosome

a)
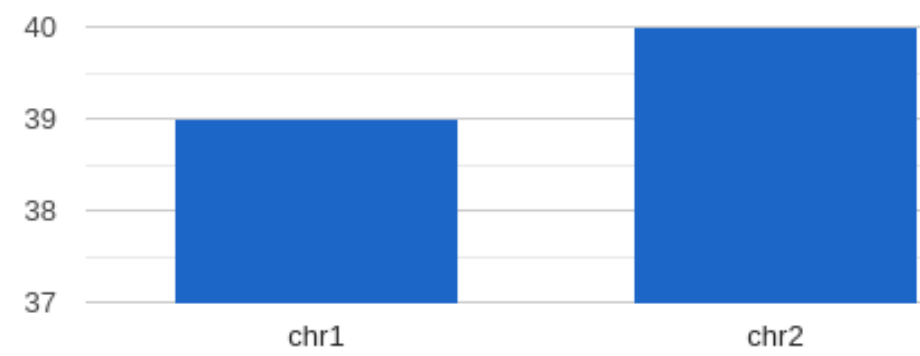

chr2

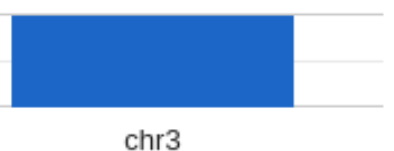

Variants by chromosome

b)

2

1

0

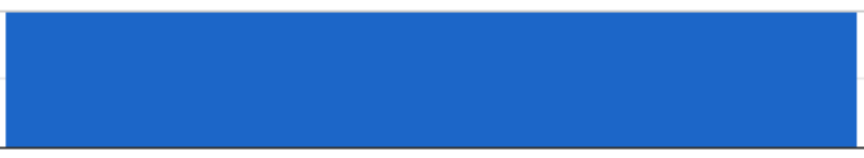


Table 1: Software dependencies required to run each component of the MeX pipeline

\begin{tabular}{|c|c|c|c|c|c|}
\hline Software & Fastp & FASTQC & ngs_te_mapper & VEP & Version Used in this Study \\
\hline Linux & $\checkmark$ & $\checkmark$ & $\checkmark$ & $\checkmark$ & 18.04 \\
\hline Conda & $\checkmark$ & $\checkmark$ & $\checkmark$ & $\checkmark$ & 4.9 .2 \\
\hline Git & $\checkmark$ & $\checkmark$ & $\checkmark$ & $\checkmark$ & 2.17 .1 \\
\hline Perl & & & $\checkmark$ & $\checkmark$ & 5.26 .2 \\
\hline Java & & $\checkmark$ & & & 11.0 .8 \\
\hline $\mathrm{C}++$ & $\checkmark$ & & & & 11 \\
\hline RepeatMasker (Smit et al. 2013) & & & $\checkmark$ & & 4.0.7 \\
\hline BEDTools (Quinlan and Hall 2010) & & & $\checkmark$ & & 2.29 .2 \\
\hline SAMTools (Li et al. 2009) & & & $\checkmark$ & & 1.9 \\
\hline BCFTools (Li et al. 2009) & & & $\checkmark$ & & 1.9 \\
\hline BWA (Li 2013) & & & $\checkmark$ & & 0.7 .17 \\
\hline
\end{tabular}

Table 2: Data dependencies required for each component of MeX pipeline

\begin{tabular}{|l|c|c|c|c|}
\hline Data files & Fastp & FASTQC & ngs_te_mapper & VEP \\
\hline Reference genome (fasta) & & & $\checkmark$ & \\
\hline Reference MGE sequences (fasta) & & & $\checkmark$ & \\
\hline $\begin{array}{l}\text { *Annotated Reference MGE sequences } \\
\text { (GFF3) }\end{array}$ & & & $\checkmark$ & \\
\hline
\end{tabular}




\begin{tabular}{|l|c|c|c|c|}
\hline Unaligned reads (single-end fastq) & $\checkmark$ & $\checkmark$ & $\checkmark$ & \\
\hline Unaligned reads (paired-end fastq) & $\checkmark$ & $\checkmark$ & $\checkmark$ & \\
\hline Identified MGE (VCF) & & & & $\checkmark$ \\
\hline
\end{tabular}


Table 1: Identified non- reference MGEs BED format

\begin{tabular}{|l|l|l|l|l|l|}
\hline Chromosome & $\begin{array}{l}\text { Start } \\
\text { Position }\end{array}$ & $\begin{array}{l}\text { End } \\
\text { Position }\end{array}$ & Information & ID & Strand Sense \\
\hline chr1 & 185100356 & 185100367 & AluYGibF9|-11|0.33|3|3|6 &. & + \\
\hline
\end{tabular}

Table 2: Identified non- reference MGEs VCF format without annotation

\begin{tabular}{|l|l|l|l|l|l|}
\hline Chromosome & Start & End & Consequence & Strand & Information \\
\hline chr1 & 185100356 & 185100367 & INS & + & $\begin{array}{l}\text { FAMILY=AluYGibF9;SUPPORTIN } \\
\text { G_READS=6;TYPE=non-reference; }\end{array}$ \\
\hline
\end{tabular}

Table 3: Identified non- reference MGEs VCF format with annotation

\begin{tabular}{|c|c|c|c|c|c|c|c|c|c|}
\hline Location & SYMBOL & Feature & Consequence & IMPACT & $\begin{array}{l}\text { VARIANT } \\
\text { CLASS }\end{array}$ & HGVSc & HGVSp & EXON & BIOTYPE \\
\hline $\begin{array}{l}\text { chr1:185100356- } \\
185100367\end{array}$ & $R N F 2$ & NM_007212.4 & $\begin{array}{l}\text { 3_prime_UTR_variant, } \\
\text { feature_elongation }\end{array}$ & MODIFIER & insertion & - & - & $7 / 7$ & protein_coding \\
\hline $\begin{array}{l}\text { chr1:185100356- } \\
185100367\end{array}$ & $R N F 2$ & XM_005245413.3 & $\begin{array}{l}\text { 3_prime_UTR_variant, } \\
\text { feature_elongation }\end{array}$ & MODIFIER & insertion & - & - & $7 / 7$ & protein_coding \\
\hline $\begin{array}{l}\text { chr1:185100356- } \\
185100367\end{array}$ & $R N F 2$ & XM_011509851.3 & $\begin{array}{l}\text { 3_prime_UTR_variant, } \\
\text { feature_elongation }\end{array}$ & MODIFIER & insertion & - & - & $7 / 7$ & protein_coding \\
\hline $\begin{array}{l}\text { chr1:185100356- } \\
185100367\end{array}$ & $R N F 2$ & XM_011509852.2 & $\begin{array}{l}\text { 3_prime_UTR_variant, } \\
\text { feature_elongation }\end{array}$ & MODIFIER & insertion & - & - & $7 / 7$ & protein_coding \\
\hline
\end{tabular}

